\title{
Investigation of the Nicole model
}

\author{
C. Adam ${ }^{a *}$, J. Sánchez-Guillén ${ }^{a * *}$, R.A. Vázquez ${ }^{a \dagger}$, and A. Wereszczyński ${ }^{b \dagger \dagger}$ \\ ${ }^{a)}$ Departamento de Fisica de Particulas, Universidad de Santiago \\ and Instituto Galego de Fisica de Altas Enerxias (IGFAE) \\ E-15782 Santiago de Compostela, Spain \\ b) Institute of Physics, Jagiellonian University, \\ Reymonta 4, 30-059 Kraków, Poland
}

\begin{abstract}
We study soliton solutions of the Nicole model - a non-linear fourdimensional field theory consisting of the $C P^{1}$ Lagrangian density to the non-integer power $\frac{3}{2}$ - using an ansatz within toroidal coordinates, which is indicated by the conformal symmetry of the static equations of motion. We calculate the soliton energies numerically and find that they grow linearly with the topological charge (Hopf index). Further we prove this behaviour to hold exactly for the ansatz. On the other hand, for the full three-dimensional system without symmetry reduction we prove a sub-linear upper bound, analogously to the case of the Faddeev-Niemi model. It follows that symmetric solitons cannot be true minimizers of the energy for sufficiently large Hopf index, again in analogy to the Faddeev-Niemi model.
\end{abstract}

\footnotetext{
*adam@fpaxp1.usc.es

**joaquin@fpaxp1.usc.es

†vazquez@fpaxp1.usc.es

${ }^{\dagger \dagger}$ wereszczynski@th.if.uj.edu.pl
} 


\section{Introduction}

In the last few years there has been rising interest in non-linear field theories which allow for the existence of ringlike, or, more generally, knotlike solitons. On the one hand, this interest is due to the fact that there may exist physical applications for such models, as is the case, for instance, for the Faddeev-Niemi model, which finds some applications both as a candidate for a low-energy effective theory for Yang-Mills theory and in condensed matter physics. On the other hand, the rising interest is related to the advance of more powerful computer facilities which allow for reliable numerical calculations of those solitons in cases when an analytical solution is not available (which happens quite often). In addition, there is some intrinsical mathematical interest in theories with knot solitons.

In the simplest case the field of the theory describes a map from onepoint compactified three-dimensional space $\mathbb{R}_{0}^{3}$ to the two-sphere $S^{2}$. $\mathbb{R}_{0}^{3}$ is topologically equivalent to the three-sphere $S^{3}$, therefore such maps are characterized by the third homotopy group of the target space $S^{2}$, which is nontrivial, $\pi_{3}\left(S^{2}\right)=\mathbb{Z}$. As a consequence, fields which describe maps $\mathbb{R}_{0}^{3} \rightarrow S^{2}$ fall into different homotopy classes, and a soliton is a field configuration which minimizes a given energy functional within a fixed homotopy class. The topological index characterizing the homotopy class is called Hopf index, the corresponding map is called a Hopf map, and the minimizers are sometimes called Hopf solitons. For details on the Hopf map we refer to Appendix A.

The probably best-known theory which allows for Hopf solitons is the Faddeev-Niemi model ([1], [2]) with Lagrangian density

$$
\mathcal{L}_{\mathrm{FN}}=\mathcal{L}_{2}-\lambda \mathcal{L}_{4}
$$

where $\lambda$ is a dimensionful coupling constant, $\mathcal{L}_{2}$ is

$$
\mathcal{L}_{2}=4 \frac{\partial_{\mu} u \partial^{\mu} \bar{u}}{(1+u \bar{u})^{2}}
$$

and $\mathcal{L}_{4}$ is

$$
\mathcal{L}_{4}=4 \frac{\left(\partial^{\mu} u \partial_{\mu} \bar{u}\right)^{2}-\left(\partial^{\mu} u \partial_{\mu} u\right)\left(\partial^{\nu} \bar{u} \partial_{\nu} \bar{u}\right)}{(1+u \bar{u})^{4}} .
$$

Further, $u$ is a complex field which parametrizes the stereographic projection of the target $S^{2}$, see Appendix A. The Faddeev-Niemi model is the $S^{2}$ 
restriction of Skyrme theory and so circumvents Derrick's theorem, because it consists of two terms such that their corresponding energies behave oppositely under a scale transformation. The existence of (static) soliton solutions for the lowest Hopf indices has been confirmed by numerical calculations ([3] $-[6])$.

There are two more models which can be constructed from the two Lagrangian densities $\mathcal{L}_{2}$ and $\mathcal{L}_{4}$ separately by choosing appropriate (noninteger) powers of these Lagrangians such that the corresponding energies are scale invariant. For $\mathcal{L}_{4}$ the appropriate choice is

$$
\mathcal{L}_{\mathrm{AFZ}}=-\left(\mathcal{L}_{4}\right)^{\frac{3}{4}}
$$

and for this model infinitely many analytic soliton solutions were found by Aratyn, Ferreira and Zimerman (=AFZ) by using an ansatz with toroidal coordinates ([7], [8]). We shall, therefore, refer to this model as the AFZ model in the sequel. The analysis of the AFZ model was carried further in ([9]), where, among other results, all the space-time and (geometric) target space symmetries of the AFZ model were determined, and, further, the use of the ansatz with toroidal coordinates was related to the conformal symmetry of the model (more precisely, of the static equations of motion). It turns out that the AFZ model has infinitely many target space symmetries and, moreover, realizes the notion of classical integrability in a rather strict sense, because the static equations of motion (e.o.m.) resulting from the ansatz with toroidal coordinates may be solved by simple integration. Let us also mention here that the model with Lagrangian $\mathcal{L}_{4}$ has the same (infinitely many) target space symmetries and, in addition, a scale invariant action, leading thereby to analytic time-dependent solutions [10].

The other model is

$$
\mathcal{L}_{\mathrm{Ni}}=\left(\mathcal{L}_{2}\right)^{\frac{3}{2}}
$$

This model has first been proposed by Nicole ([11]), and it was shown in the same paper that the simplest Hopf map with Hopf index 1 is a soliton solution for this model. We, therefore, call this model the Nicole $(=\mathrm{Ni})$ model in this paper. This model shares the conformal symmetry with the AFZ model, therefore, again the ansatz with toroidal coordinates may be used to simplify the static e.o.m. (to reduce them to an ordinary differential equation). However, the Nicole model only has the obvious symmetries the conformal base space symmetries (in the static case) and the modular 
target space symmetries, see [12]. Consequently, the e.o.m. are no longer integrable, and the solutions are no longer available in closed, analytic form (except for the simplest case with Hopf index one ${ }^{1}$ ). It is the main purpose of this paper to analyse these soliton solutions with higher Hopf index by developing the analytical treatment as far as possible and by performing numerical calculations where it is necessary.

The AFZ and Nicole models currently do not have direct physical applications, but they can serve as useful test labors for the understanding of generic features of models with knot solitons. This is all the more true as analytic results - already for solitons, but even more so for time-dependent problems like soliton scattering - are notoriously difficult to obtain for realistic models like the Faddeev-Niemi model. Both the AFZ and the Nicole model are much easier to treat, and for time dependent problems the Nicole model even has one advantage over the AFZ model. The infinitely many symmetries of the former - which provide its integrability on the one hand - imply that the moduli space for multi-soliton configurations is infinite dimensional. This most likely makes an adiabatic treatment of soliton scattering problematic. On the other hand, that problem is absent for the Nicole model which may, therefore, probably serve as a natural test labor for an adiabatic study of the scattering of knot-like solitons. A further issue which may be useful for the study of time dependent problems is provided by the observation that all field configurations within the toroidal ansatz belong to an integrable subsector of the Nicole model (i.e., to a sector with infinitely many conservad currents), see Section 3.

The paper is organized as follows. In Section 2 we provide the static e.o.m. of the Nicole model as well as the non-linear ODE resulting from the ansatz with toroidal coordinates. Then we analyse the latter equation in detail, establishing some properties analytically. In a next step we calculate the energies of the corresponding solitons numerically with high precision. We provide all energies for solitons with sufficiently small Hopf index. Further, we calculate some energies for larger Hopf index in order to establish some generic behaviour. We prove that this generic behaviour is in accordance with exact energy estimates for the ansatz in toroidal coordinates.

In Section 3 we show that for all the solutions of Section 2 there exist infinitely many conserved currents, that is to say, all these field configurations

\footnotetext{
${ }^{1}$ Exact solitons with higher Hopf index have been found in modified Nicole models [13]
} 
belong to an integrable subsector of the Nicole model.

In Section 4.1 we prove an upper bound for the energies of field configurations with given Hopf index, of the type $E_{Q} \leq C_{2} Q^{\frac{3}{4}}$, and provide an explicit value for the constant $C_{2}$. In Section 4.2 we derive an analogous bound for the AFZ and Faddeev-Niemi model, which is easily achieved with the help of the results of Section 4.1. The discussion of the possibility to find a corresponding lower bound $E_{Q} \geq C_{1} Q^{\frac{3}{4}}$ for the Nicole model, analogously to the Vakulenko-Kapitansky bound for the Faddeev-Niemi model, is presented in Appendix C. We show where a Vakulenko-Kapitansky type proof needs to be refined for the Nicole (and also for the AFZ) model and leave this lower bound as a conjecture for the moment.

Section 5 contains our conclusions, where we comment on the relevance of our results for issues like stability, and on the relation to the corresponding results for the other two models (Faddeev-Niemi and AFZ). In Appendix A we collect useful results and facts about Hopf maps which we use throughout the paper. Appendix B contains some proofs which we need in the main sections. In Appendix $\mathrm{C}$ we discuss the lower energy bound.

\section{Solitons in the Nicole model}

\subsection{Equation of motion}

The static e.o.m. for the Nicole model (5) is

$$
\frac{1}{2}\left(u_{j k} \bar{u}_{j} u_{k}+\bar{u}_{j k} u_{j} u_{k}\right)+u_{j} \bar{u}_{j} u_{k k}-\frac{u_{j} \bar{u}_{j}}{1+u \bar{u}}\left(u_{k} \bar{u}_{k} u+3 u_{k} u_{k} \bar{u}\right)=0
$$

where $u_{k} \equiv \partial_{\mu} u$ etc., and the Einstein summation convention is understood. We now introduce toroidal coordinates $(\eta, \xi, \varphi)$ via

$$
\begin{aligned}
& x=q^{-1} \sinh \eta \cos \varphi, \quad y=q^{-1} \sinh \eta \sin \varphi \\
& z=q^{-1} \sin \xi ; \quad q=\cosh \eta-\cos \xi
\end{aligned}
$$

Further, we choose for $u$ the ansatz

$$
u=f(\eta) e^{i m \varphi+i n \xi} \quad, \quad m, n \in \mathbb{Z}
$$

which is compatible with the e.o.m. for the Nicole model as well as for the

AFZ model. The reason for this ansatz may, in fact, be understood from 
the symmetries of the model. More precisely, the presence of the conformal symmetry on base space implies that the ansatz (8) is an "educated guess" for a solution to Eq. (6) in the sense of the Lie theory of symmetry. That is to say, if we choose a rotation about the $z$ axis and a certain combination of proper conformal transformation along the $z$ axis and translation along the $z$ axis as a maximal set of two commuting base space transformations, then the corresponding infinitesimal symmetry generators (vectors $\mathbf{v}^{i}$ ) are precisely given by the tangent vectors along $\varphi$ and $\xi, \mathbf{v}^{1}=\partial_{\varphi}$, and $\mathbf{v}^{2}=\partial_{\xi}$. The ansatz (8) is invariant under a combination of these base space transformations and phase transformations of the target space variable $u$, i.e., under the action of the vector fields $\tilde{\mathbf{v}}^{1}=\partial_{\varphi}-i m u \partial_{u}$ and $\tilde{\mathbf{v}}^{2}=\partial_{\xi}-i n u \partial_{u}$, which provides precisely the educated guess according to Lie. A concise discussion of these points can be found in Ref. [9], where the symmetries of the AFZ model are discussed in great detail.

We need the gradient in toroidal coordinates

$$
\nabla=(\nabla \eta) \partial_{\eta}+(\nabla \xi) \partial_{\xi}+(\nabla \varphi) \partial_{\varphi}=q\left(\hat{e}_{\eta} \partial_{\eta}+\hat{e}_{\xi} \partial_{\xi}+\frac{1}{\sinh \eta} \hat{e}_{\varphi} \partial_{\varphi}\right)
$$

where $\left(\hat{e}_{\eta}, \hat{e}_{\xi}, \hat{e}_{\varphi}\right)$ form an orthonormal frame in $\mathbb{R}^{3}$. Further we need the relations

$$
\nabla \cdot \hat{e}_{\eta}=-\sinh \eta+\frac{1-\cosh \eta \cos \xi}{\sinh \eta}, \quad \nabla \cdot \hat{e}_{\xi}=-2 \sin \xi, \quad \nabla \cdot \hat{e}_{\varphi}=0 .
$$

Inserting now the ansatz (8) into the static e.o.m. (6) we find, after a straightforward calculation, that the ansatz is indeed compatible with the static e.o.m. and that $f$ has to obey the non-linear ODE

$$
\begin{gathered}
2 f^{\prime \prime} f^{\prime 2}+f^{\prime \prime} f^{2}\left(n^{2}+\frac{m^{2}}{\sinh ^{2} \eta}\right)+\left(f^{\prime 3}+n^{2} f^{\prime} f^{2}\right) \frac{\cosh \eta}{\sinh \eta}-f^{3}\left(n^{2}+\frac{m^{2}}{\sinh ^{2} \eta}\right)^{2}- \\
-f \frac{f^{\prime 2}+f^{2}\left(n^{2}+\frac{m^{2}}{\sinh ^{2} \eta}\right)}{1+f^{2}}\left(4 f^{\prime 2}-2 f^{2}\left(n^{2}+\frac{m^{2}}{\sinh ^{2} \eta}\right)\right)=0
\end{gathered}
$$

where $f^{\prime} \equiv \partial_{\eta} f$ etc. It may be checked without difficulty that the simplest Hopf map

$$
u=\sinh \eta e^{i \xi+i \varphi} \Rightarrow f=\sinh \eta, \quad m=n=1
$$


indeed solves the above equation.

For technical reasons it is preferable to introduce the new variable

$$
t \equiv \sinh \eta
$$

in terms of which the above e.o.m. can be expressed as a pure polynomial in the independent and dependent variables $t, f, f_{t}, f_{t t}$,

$$
\begin{gathered}
F\left(t, f, f_{t}, f_{t t}\right) \equiv \\
t^{2}\left(1+t^{2}\right)\left(1+f^{2}\right)\left(2 t^{2}\left(1+t^{2}\right) f_{t}^{2}+\left(n^{2} t^{2}+m^{2}\right) f^{2}\right) f_{t t}-4 t^{4}\left(1+t^{2}\right)^{2} f f_{t}^{4}+ \\
+t^{3}\left(1+3 t^{2}\right)\left(1+t^{2}\right)\left(1+f^{2}\right) f_{t}^{3}-2 t^{2}\left(1+t^{2}\right)\left(n^{2} t^{2}+m^{2}\right) f^{3} f_{t}^{2}+ \\
+t^{3}\left(m^{2}+n^{2}\left(1+2 t^{2}\right)\right)\left(1+f^{2}\right) f^{2} f_{t}-\left(n^{2} t^{2}+m^{2}\right)^{2} f^{3}\left(1-f^{2}\right)=0
\end{gathered}
$$

where $f_{t} \equiv \partial_{t} f$ etc. Further, we need the energy functional $E[f]$ which results for the ansatz (8) after the integration w.r.t. the variables $\xi, \varphi$. With

$$
d V \equiv d^{3} r=q^{-3} \sinh \eta d \eta d \xi d \varphi
$$

we find

$$
\begin{aligned}
E[f] & =32 \pi^{2} \int_{0}^{\infty} d \eta \sinh \eta \frac{\left(f_{\eta}^{2}+f^{2}\left(n^{2}+\frac{m^{2}}{\sinh ^{2} \eta}\right)\right)^{\frac{3}{2}}}{\left(1+f^{2}\right)^{3}} \\
& =32 \pi^{2} \int_{0}^{\infty} d t t\left(1+t^{2}\right) \frac{\left(f_{t}^{2}+\left(n^{2}+\frac{m^{2}}{t^{2}}\right) \frac{f^{2}}{1+t^{2}}\right)^{\frac{3}{2}}}{\left(1+f^{2}\right)^{3}}
\end{aligned}
$$

For $n=m=1$ for the solution $f(t)=t$ the energy may be calculated analytically,

$$
E=32 \pi^{2} 2^{\frac{3}{2}} \int_{0}^{\infty} \frac{d t t}{\left(1+t^{2}\right)^{2}}=32 \pi^{2} \sqrt{2} .
$$

If we calculate the energy for the field configuration $f(t)=c t$ instead, we find

$$
E=32 \pi^{2} \sqrt{2} \frac{1}{2}\left(c+\frac{1}{c}\right)
$$

which certainly has a minimum at $c=1$ but also shows clearly that the onesoliton sector is indeed separated from the trivial sector (with Hopf index zero) by an infinite energy barrier. 
Next we have to fix the boundary conditions which $f$ has to obey. The $u$ of Eq. (8) has to take values in the whole target space $\mathbb{C}_{0}$ in order to be a Hopf map. This implies that $f$ has to take values in the whole positive real numbers, including zero, i.e., in the whole $\mathbb{R}_{+0}$. It follows that the possible boundary conditions on $f$ are restricted to one of the two following options. Either $f(0)=0, f(\infty)=\infty$ or $f(0)=\infty, f(\infty)=0$. The reason for this is that the values $f=0$ and $f=\infty$ correspond to the north and south pole of the target space $S^{2}$, respectively. Consequently, their pre-images in the base space $\mathbb{R}_{0}^{3}$ must be one-dimensional objects (closed lines). But the only two values $\eta=\eta_{0}=$ const (or $t=t_{0}=$ const) which correspond to lines rather than surfaces (tori) are the values $\eta=0(t=0)$ and $\eta=\infty(t=\infty)$. Therefore we assume

$$
f(\eta=0)=f(t=0)=0, \quad f(\eta=\infty)=f(t=\infty)=\infty
$$

which is general, because the other option is related to this one by a symmetry transformation.

\subsection{Asymptotic behaviour and numerical evaluation}

In a next step we want to determine the asymptotic behaviour of the function $f(t)$ for small and large values of $t$ from the differential equation (14). For small $t$ we assume that $f(t) \sim t^{\alpha}+o\left(t^{\beta}\right)$ where $\beta>\alpha>0$. From Eq. (14) we get

$$
F\left(t, f, f_{t}, f_{t t}\right) \sim\left(2 \alpha^{4}-\alpha^{3}+\alpha^{2} m^{2}-\alpha m^{2}-m^{4}\right) t^{3 \alpha}+o\left(t^{3 \alpha+2}\right)+o\left(t^{3 \beta}\right) \equiv 0
$$

The condition that the leading (i.e., smallest) order $t^{3 \alpha}$ is absent therefore leads to

$$
2 \alpha^{4}-\alpha^{3}+\alpha^{2} m^{2}-\alpha m^{2}-m^{4}=0
$$

which has four solutions for $\alpha$ of which only one is acceptable (real and positive), namely

$$
\alpha_{m} \equiv \frac{1}{4}\left(1+\sqrt{8 m^{2}+1}\right)
$$

In the same fashion we may determine the subleading (higher order) contributions to $f$ for small $t$ in an iterative manner. We find

$$
f=t^{\alpha_{m}} P^{(0)}\left(t, t^{\alpha_{m}}\right) \quad \text { for } \quad t<<1
$$


where $P^{(0)}\left(t, t^{\alpha_{m}}\right)$ is a polynomial of its arguments, which is determined up to a multiplicative constant,

$$
P^{(0)}\left(t, t^{\alpha_{m}}\right)=\sum_{k, l=0}^{\infty} c_{k l}^{(0)} t^{k} t^{l \alpha_{m}} .
$$

Here it is possible to determine the higher coefficients $c_{k l}^{(0)}$ in terms of $m, n$ and $c_{00}^{(0)}$. Unfortunately, it is not possible to determine $c_{00}^{(0)}$, i.e., an overall constant remains undetermined by this asymptotic analysis.

We repeat the same asymptotic analysis for large values of $t$. We assume that $f(t) \sim t^{\alpha}+o\left(t^{\beta}\right)$ where now $\alpha>\beta>0$ and find

$F\left(t, f, f_{t}, f_{t t}\right) \sim\left(2 \alpha^{4}-\alpha^{3}+\alpha^{2} n^{2}-\alpha n^{2}-n^{4}\right) t^{5 \alpha+4}+o\left(t^{5 \alpha+2}\right)+o\left(t^{5 \beta+4}\right) \equiv 0$

and therefore the same condition for $\alpha$ like for small $\mathrm{t}$ with the only replacement $m \rightarrow n$. We may again determine $f$ asymptotically up to an overall constant,

$$
f=t^{\alpha_{n}} P^{(\infty)}\left(t^{-1}, t^{-\alpha_{n}}\right) \quad \text { for } \quad t>>1
$$

where

$$
P^{(\infty)}\left(t^{-1}, t^{-\alpha_{n}}\right)=\sum_{k, l=0}^{\infty} c_{k l}^{(\infty)} t^{-k} t^{-l \alpha_{n}}
$$

and again the higher coefficients $c_{k l}^{(\infty)}$ are determined in terms of $m, n$ and $c_{00}^{(0)}$. Again, $c_{00}^{(\infty)}$ remains undetermined.

Next we turn to the numerical evaluation of the soliton energies for general $m, n$. For this purpose it is preferable to minimize the energy functional (16). The problem is that standard evolution procedures for the differential equation (14) cannot be used because of the singular nature of this equation at $t=0$. We proceed as follows with the minimisation. We first factorize the leading behaviour, e.g., we choose

$$
f(t) \equiv t^{\alpha_{m}}\left(1+t^{2}\right)^{\frac{\alpha_{n}-\alpha_{m}}{2}} g(t)
$$

where $g$ obeys

$$
g>0 \quad \forall \quad t, \quad g(0)=g_{0}, \quad g(\infty)=g_{\infty}
$$


Then we make a finite parameter ansatz for $g$ of the form

$$
g(t)=\frac{g_{0}+g_{\infty} t^{2}}{1+t^{2}} \frac{1+\sum_{i=1}^{k} a_{i} t^{2 i}+t^{k+2}}{1+\sum_{j=1}^{k} b_{j} t^{2 j}+t^{k+2}}
$$

where $g_{0}, g_{\infty}, a_{i}$ and $b_{j}$ are the parameters with respect to which we minimize the energy functional (16). For each value of $m, n$ we increase the number of parameters (i.e., the integer number $k$ ), until we reach stability (i.e., until the energies do not change further with the increase of the number of parameters). We do not choose a full numerical minimization of the energy functional for the following reason. It turns out that the leading behaviour (i.e., $g(t)=$ const), already provides rather good results for the corresponding energies. This implies that the energy functional, when viewed as a functional of $g$, is rather shallow. As a consequence, a full numerical minimisation which implements the derivatives numerically has the tendency to produce false minima unless the numerical minimisation grid is chosen very finely (and consumes a lot of computer time). On the other hand, within our finite parameter ansatz we are able to perform the derivatives analytically, and therefore this problem is absent.

We show our numerical results in Table 1 for all $(m, n)=(1,1) \ldots(5,5)$ as well as for some higher values. Further we plot the energies versus the Hopf index $Q=m n$ in Fig. 1. From our results it is obvious that the energies grow linearly in $Q$ to a very good accuracy. More precisely, the energies for $m=n$ lie on a straight line in Fig. 1 almost exactly. The energies for $m \neq n$ lie slightly above, whereas no energy lies below this line. It is in fact possible to prove the linear growth of energies with the Hopf charge for solutions of the ansatz (8). This we show for the simplest case $m=n$ in the next subsection.

On the other hand, for the full model the sublinear upper bound $E \leq$ $C_{2} Q^{\frac{3}{4}}$ holds, which we prove in Section 4. It follows that the solutions we found for the symmetric ansatz (8) cannot be true solitons, i.e., global minima of topological sectors with fixed $Q$ for sufficiently large values of $Q$. This is akin to the situation in the Faddeev-Niemi model and differs from the situation in the AFZ model (see our discussion in Section 5).

\subsection{Upper and lower bounds for the torus ansatz}

In this subsection we prove the bounds $c_{1} Q \leq E\left[f_{m, n}\right]$ and, for the special case $m=n, E\left[f_{m, m}\right] \leq c_{2} m^{2}$ (with explicit values for the constants $c_{1}$ and 


\begin{tabular}{||c|r|c||r|r|c||}
\hline$n$ & $m$ & $E^{*}$ & $n$ & $m$ & $E^{*}$ \\
\hline 1 & 1 & 1.41362193 & 4 & 1 & 6.52450266 \\
1 & 2 & 2.62615091 & 4 & 2 & 9.03384901 \\
1 & 3 & 4.13338723 & 4 & 3 & 12.7436624 \\
1 & 4 & 5.37349409 & 4 & 4 & 16.789796 \\
1 & 5 & 7.54150789 & 4 & 5 & 20.886948 \\
2 & 1 & 2.64753548 & 5 & 1 & 9.48680075 \\
2 & 2 & 4.52249973 & 5 & 2 & 11.8901415 \\
2 & 3 & 6.62925943 & 5 & 3 & 15.9786399 \\
2 & 4 & 8.85370929 & 5 & 4 & 20.8937915 \\
2 & 5 & 10.9513184 & 5 & 5 & 25.975942 \\
3 & 1 & 4.28232266 & 6 & 6 & 37.2017869 \\
3 & 2 & 6.64941801 & 8 & 8 & 65.771712 \\
3 & 3 & 9.64059435 & 9 & 9 & 83.11675 \\
3 & 4 & 12.7307136 & 10 & 10 & 102.501125 \\
3 & 5 & 15.8672836 & 20 & 20 & 408.581244 \\
& & & 5 & 10 & 51.8990747 \\
& & & 6 & 15 & 96.0585933 \\
& & & 14 & 7 & 103.670713 \\
\hline
\end{tabular}

Table 1: The rescaled energies $E^{*}=\left(32 \pi^{2}\right)^{-1} E$ of solutions for selected values of $m$ and $n$. 
$c_{2}$ ) in order to further support our numerical results. Here $E[f]$ is the energy functional (16) and $f_{m, n}$ is the corresponding solution of the equation of motion. We first prove the lower bound. We use the inequality

$$
(a+b)^{\frac{3}{2}} \geq \frac{3 \sqrt{3}}{2} a^{\frac{1}{2}} b \quad \text { for } \quad a, b \geq 0
$$

which we prove in Appendix B (here $\frac{3 \sqrt{3}}{2}$ is an optimal value). We choose $a=f_{t}^{2}$ and $b=\left(n^{2}+m^{2} / t^{2}\right) f^{2} /\left(1+t^{2}\right)$ and introduce

$$
Q=m n, \quad \mu=\frac{m}{n}
$$

to get

$$
\begin{aligned}
E[f] & =32 \pi^{2} \int_{0}^{\infty} d t t\left(1+t^{2}\right) \frac{\left(f_{t}^{2}+Q\left(\frac{1}{\mu}+\frac{\mu}{t^{2}}\right) \frac{f^{2}}{1+t^{2}}\right)^{\frac{3}{2}}}{\left(1+f^{2}\right)^{3}} \\
& \geq 32 \pi^{2} Q \frac{3 \sqrt{3}}{2} \int_{0}^{\infty} d t\left(\frac{t}{\mu}+\frac{\mu}{t}\right)\left|f_{t}\right| \frac{f^{2}}{\left(1+f^{2}\right)^{3}} \\
& \geq 32 \pi^{2} Q 3 \sqrt{3} \int_{0}^{\infty} d t f_{t} \frac{f^{2}}{\left(1+f^{2}\right)^{3}} \equiv \tilde{E}[f]
\end{aligned}
$$

where we used $(t / \mu+\mu / t) \geq 2$. Up to now we have in fact used inequalities for the energy densities, i.e., these inequalities hold for given, fixed functions $f$. However, if we evaluate the last expression for its minimizer $f_{\text {min }}$ then the inequality holds for arbitrary $f$ and, consequently, for the solutions $f_{m, n}$. The minimization of the last expression is simplified by the observation that it is a total derivative. Therefore it is minimized for all $f$ which obey the required boundary conditions $f(0)=0$ and $f(\infty)=\infty$. Explicitly we get

$$
\begin{aligned}
\tilde{E}[f] & =32 \pi^{2} Q 3 \sqrt{3}\left[-\frac{f}{4\left(1+f^{2}\right)^{2}}+\frac{f}{8\left(1+f^{2}\right)}+\frac{1}{8} \arctan f\right]_{f=0}^{f=\infty} \\
& =32 \pi^{2} Q 3 \sqrt{3} \frac{\pi}{16}=1.02 \times 32 \pi^{2} Q
\end{aligned}
$$

Comparing with Table 1, we see that for larger values of $m, n$ our lower bound gets very close to the numerical values, especially for $m=n$ (e.g., better than one per cent for $m=n=10$ or $m=n=20$ ). This demonstrates the very good accuracy of our numerical results. 
For the derivation of an upper bound we restrict to the simpler case $m=n$. We simply insert a trial function $f_{m, m}^{\mathrm{tr}}$ into the energy functional $E[f]$. Concretely we choose $f_{m, m}^{\mathrm{tr}}=t^{m}$ and get

$$
E\left[t^{m}\right]=32 \pi^{2} \int_{0}^{\infty} d t t\left(1+t^{2}\right) \frac{m^{3} t^{3 m-3} 2^{\frac{3}{2}}}{\left(1+t^{2 m}\right)^{3}}
$$

and, with the substitution $u=t^{m}$,

$$
\begin{aligned}
E\left[t^{m}\right] & =32 \pi^{2} m^{2} 2^{\frac{3}{2}} \int_{0}^{\infty} d u\left(u^{\frac{1}{m}}+u^{-\frac{1}{m}}\right) \frac{u^{2}}{\left(1+u^{2}\right)^{3}} \\
& =32 \pi^{2} m^{2} 2^{\frac{3}{2}} \frac{1}{8}\left(1-\frac{1}{m^{2}}\right) \frac{\pi}{\cos \frac{\pi}{2 m}} .
\end{aligned}
$$

The function $\left(1-1 / m^{2}\right) /\left(\cos \frac{\pi}{2 m}\right)$ is a monotonously decreasing function in $m$ and may, therefore, be estimated by its value at $m=2$ for the range $m \geq 2$. With

$$
\left(1-\frac{1}{4}\right) \frac{1}{\cos \frac{\pi}{4}}=\frac{3}{4} \sqrt{2}
$$

we therefore get

$$
E\left[t^{m}\right] \leq 32 \pi^{2} m^{2} \frac{3 \pi}{8}=1.18 \times 32 \pi^{2} m^{2} \quad \text { for } \quad m \geq 2
$$

which, for large $m$, is about 15 percent above the numerical value. By using Eq. (36) instead of the order $m^{2}$ estimate (38), we get slightly better results which, for large $m$, are less than 10 percent above the numerical values.

In short, we established the bounds $c_{1} m^{2} \leq E\left[f_{m, m}\right] \leq c_{2} m^{2}$ with $c_{1}=$ $1.02 \times 32 \pi^{2}$ and $c_{2}=1.18 \times 32 \pi^{2}$, as announced.

\section{Infinitely many conserved currents}

In this section we want to demonstrate that the ansatz (8) belongs to a subsector of the Nicole model with infinitely many conserved currents for arbitrary profile function $f(\eta)$. The integrability condition defining this subsector exists for a wide class of models, and discussing it for the whole class does not complicate matters (for a more detailed discussion, and especially for the geometric meaning of the integrability condition, we refer to [14]; the 
concept of higher dimensional integrability, which is at the basis of these discussions, was introduced in [15]). Therefore, we start with the general class of Lagrangian densities

$$
\mathcal{L}\left(u, \bar{u}, u_{\mu}, \bar{u}_{\mu}\right)=F(a, b, c)
$$

where

$$
a=u \bar{u}, \quad b=u_{\mu} \bar{u}^{\mu}, \quad c=\left(u_{\mu} \bar{u}^{\mu}\right)^{2}-u_{\mu}^{2} \bar{u}_{\nu}^{2}
$$

and $F$ is an arbitrary real function of its arguments. Obviously, the Nicole model belongs to this class, $\mathcal{L}_{\mathrm{Ni}}=\left[4(1+a)^{-1} b\right]^{\frac{3}{2}}$.

Further, we define the currents

$$
K^{\mu}=h(a) \bar{\Pi}^{\mu}
$$

where $h$ is an arbitrary given real function of its argument, and $\Pi^{\mu}$ and $\bar{\Pi}^{\mu}$ are the conjugate four-momenta of $u$ and $\bar{u}$, i.e.,

$$
\Pi_{\mu} \equiv \mathcal{L}_{u^{\mu}}=\bar{u}^{\mu} F_{b}+2\left(u^{\lambda} \bar{u}_{\lambda} \bar{u}_{\mu}-\bar{u}_{\lambda}^{2} u_{\mu}\right) F_{c} .
$$

Finally, we define the infinitely many Noether currents

$$
J_{\mu}^{G}=i\left(G_{u} K_{\mu}-G_{\bar{u}} \bar{K}_{\mu}\right)
$$

where $G$ is an arbitrary real function of $u$ and $\bar{u}$, and $G_{u} \equiv \partial_{u} G$.

Now we want to study the conditions which one has to impose in order to make the divergence $\partial^{\mu} J_{\mu}^{G}$ vanish. A simple calculation reveals

$$
\begin{aligned}
\partial^{\mu} J_{\mu}^{G}= & i h\left(\left[\left(\frac{h^{\prime}}{h} \bar{u} G_{u}+G_{u u}\right) u_{\mu}^{2}-\left(\frac{h^{\prime}}{h} u G_{\bar{u}}+G_{\bar{u} \bar{u}}\right) \bar{u}_{\mu}^{2}\right] F_{b}\right. \\
& \left.+\left(u G_{u}-\bar{u} G_{\bar{u}}\right)\left[\frac{h^{\prime}}{h}\left(b F_{b}+2 c F_{c}\right)+F_{a}\right]\right)
\end{aligned}
$$

where the prime denotes the derivative with respect to $a$.

The second term at the r.h.s. of Eq. (44) certainly vanishes if

$$
u G_{u}-\bar{u} G_{\bar{u}}=0
$$

with the general solution

$$
G(u, \bar{u})=\tilde{G}(u \bar{u}) \equiv \tilde{G}(a) .
$$


Without taking condition (46) into account, the first term on the r.h.s. of Eq. (44) vanishes for the integrability condition

$$
u_{\mu}^{2}=0
$$

i.e., the complex eikonal equation (it also vanishes for some more complicated integrability conditions which we do not discuss here, see Refs. [16], [17]). However, by using condition (46) we may re-express the first term like

$$
\left(h^{\prime} G^{\prime}+h G^{\prime \prime}\right) F_{b}\left[\bar{u}^{2} u_{\mu}^{2}-u^{2} \bar{u}_{\mu}^{2}\right]
$$

and, therefore, we find, instead of the complex eikonal equation, the weaker integrability condition

$$
\bar{u}^{2} u_{\mu}^{2}-u^{2} \bar{u}_{\mu}^{2}=0 .
$$

The meaning of this condition becomes especially transparent when we reexpress $u$ in terms of its modulus and phase like

$$
u=\exp (\Sigma+i \Lambda)
$$

Then the complex eikonal equation is equivalent to the two real equations

$$
\Sigma_{\mu}^{2}=\Lambda_{\mu}^{2}
$$

and

$$
\Sigma^{\mu} \Lambda_{\mu}=0
$$

whereas the weaker condition (49) becomes Eq. (52) alone or, for timeindependent $u$,

$$
(\nabla \Sigma) \cdot(\nabla \Lambda)=0
$$

Remark: The condition $G(u, \bar{u})=\tilde{G}(u \bar{u})$, Eq. (46), restricts the space of allowed $G$ to a subspace which is still infinite-dimensional, therefore the integrability condition (49) really defines a subsector with infinitely many conserved currents.

Remark: The ansatz (8) obeys the integrability condition (53) for arbitrary profile function $f(\eta)$, as is obvious from the orthonormality of the basis vectors $\left(\hat{e}_{\eta}, \hat{e}_{\xi}, \hat{e}_{\varphi}\right)$. Therefore, all our solutions of Section 2 really belong to the subsector of the Nicole model defined by condition (49) with infinitely many conserved currents. This is in contrast to the complex eikonal equation 
(47), which leads to a non-linear ODE for the profile function which is not compatible with the Nicole model field equation except for the simplest Hopf map $m=n=1$, see [18].

Remark: Both condition (46) and the integrability condition (49) do not restrict the Lagrangian density, therefore the corresponding integrable sub-

sectors with infinitely many conservation laws exist for all Lagrangians of the type (39). So they may be of interest for other models like, e.g., the Faddeev-Niemi model.

\section{Energy estimates for the full model}

In this section we study upper bounds on the energies of solitons with a given Hopf index, of the type $E_{Q} \leq C_{2} Q^{\frac{3}{4}}$. In Subsection 4.1 we prove the upper bound $E_{Q} \leq C_{2} Q^{\frac{3}{4}}$ by following the method used in [19] for the FaddeevNiemi model. Further, we provide an explicit value for the constant $C_{2}$. In Subsection 4.2, we briefly derive the analogous upper bounds for the AFZ and the Faddeev-Niemi models, again with explicit values for the constants $C_{2}$. They easily follow from our results from Subsection 4.1. The issue of a lower bound $C_{1} Q^{\frac{3}{4}} \leq E_{Q}$ is discussed in Appendix C. There we show where a naive Vakulenko-Kapitansky type proof does not work and needs some refinement in our case, leaving open for the moment the problem of rigorously establishing such a lower bound.

\subsection{The estimate $E_{Q} \leq C_{2} Q^{3 / 4}$}

In this section we use the unit vector $\vec{n}$ to denote Hopf maps, because this has some technical advantages (components of the vector $\vec{n}$ can be easily estimated, whereas the complex function $u$ may take arbitrary values). In our proof we will closely follow the method used in [19] for the proof of an analogous result for the Faddeev-Niemi model. However, we will use explicit expressions for certain functions instead of using just their existence, which enables us to provide an explicit value for the constant $C_{2}$ in our estimate.

For a Hopf index which is a square, $Q=l^{2}$, a Hopf map is constructed in [19] which is a composition of a Hopf map $\vec{n}(\vec{r})$ with Hopf index 1 and a map $\vec{N}(\vec{n}): S^{2} \rightarrow S^{2}$ with winding number $w=l$. The Hopf map $\vec{n}(\vec{r})$ is constructed such that it varies smoothly from $\vec{n}=(0,0,1)$ at $\vec{r}=0$ to 
$\vec{n}=(0,0,-1)$ at $|\vec{r}|=R$ and remains at this value for $r>R$. This implies, by Eq. (114) of Appendix A, that it has indeed Hopf index 1. Further, the gradient $\left|\nabla^{\mathbb{R}^{3}} \vec{n}\right|$ may be estimated like $\left|\nabla^{\mathbb{R}^{3}} \vec{n}\right| \leq c / R$ for $r \leq R$ and by $\left|\nabla^{\mathbb{R}^{3}} \vec{n}\right|=0$ for $r>R$ (here and below $c$ denotes an unspecified constant). The map $\vec{N}(\vec{n}): S^{2} \rightarrow S^{2}$ is constructed such that $\vec{N}$ varies smoothly over $l$ nonintersecting geodesic discs covering the base $S^{2}$, where $\vec{N}=(0,0,1)$ in the center of each disc and $\vec{N}=(0,0,-1)$ at the boundary of each disc and between the discs. Each disc contributes a winding number 1 for the map $\vec{N}(\vec{n})$, therefore the total winding number is $l$. Further, the geodesic radius of each disc can be chosen (not larger than) $R_{g}=c l^{-\frac{1}{2}}$ in order to be able to put $l$ nonintersecting discs on one unit $S^{2}$. The gradient may be again estimated by the inverse (geodesic) radius, $\left|\nabla^{S^{2}} \vec{N}(\vec{n})\right| \leq c l^{\frac{1}{2}}$, and with the help of the chain rule one finds for the gradient of $\vec{N}$ that $\left|\nabla^{\mathbb{R}_{0}^{3}} \vec{N}(\vec{n}(\vec{r}))\right| \leq c R^{-1} l^{\frac{1}{2}}$ and for the energy density $\mathcal{E}_{\mathrm{Ni}}(\vec{r}) \sim\left|\nabla^{\mathbb{R}_{0}^{3}} \vec{N}\right|^{3} \leq c R^{-3} l^{\frac{3}{2}}$. The energy $E_{\mathrm{Ni}}$, which is the integral over all space of $\mathcal{E}_{\mathrm{Ni}}(\vec{r})$, may in the case at hand be calculated by integrating over the ball $B^{R}$ of radius $R$ with the result that $E_{\mathrm{Ni}} \leq c l^{\frac{3}{2}} \equiv c Q^{\frac{3}{4}}$ which is the announced estimate for $Q=l^{2}$.

Before rederiving this result in more detail and with explicit choices for the maps described above in rather general terms, it may be useful to have a geometric picture of the above compostion of maps. In fact, the map $\vec{N}(\vec{n}(\vec{r}))$ describes $l$ nonintersecting, full tori confined to the ball $B^{R}$, where, in addition, each full torus is linked with all other tori. In the center of each torus there is a closed line along which $\vec{N}$ takes the value $\vec{N}=(0,0,1)$. From the center to the surface of each full torus, $\vec{N}$ varies smoothly from $\vec{N}=(0,0,1)$ to $\vec{N}=(0,0,-1)$. At the surface of each full torus (which has the topology of the two-torus $T^{2}$ ) as well as in the space between the tori and outside the ball $B^{R}, \vec{N}$ remains at the value $\vec{N}=(0,0,-1)$.

Now for the detailed derivation, where we still assume $Q=l^{2}$ for the moment. For the map $\vec{n}(\vec{r})=(\sin \vartheta \cos \phi, \sin \vartheta \sin \phi, \cos \vartheta)$ with Hopf index 1 we assume that it has cylindrical symmetry, as in Eq. (118) of Appendix A. For the profile function $\rho(r)$ we want to find a choice which varies as smoothly as possible from 0 to $\pi$ as $r$ varies from 0 to $R$, in order to be able to estimate it with a number as small as possible. The smoothest choice $\rho=\pi r / R$ for $r \leq R$ and $\rho=\pi$ for $r>R$ does not have a continuous first derivative at $r=R$, which we need for the energy density, but there is a 
simple generalization which does have one. We choose

$$
\rho_{\epsilon}(r)=\pi F_{\epsilon}(x), \quad x \equiv \frac{r}{R}
$$

where

$$
\begin{aligned}
& F_{\epsilon}(x)=F_{\epsilon}^{<}(x) \equiv \frac{2}{2-\epsilon} x \text { for } 0 \leq x \leq 1-\epsilon \\
& F_{\epsilon}(x)=F_{\epsilon}^{>}(x) \equiv 1-\frac{1}{\epsilon(2-\epsilon)}(x-1)^{2} \text { for } 1-\epsilon<x \leq 1 \\
& F_{\epsilon}(x)=1 \text { for } x>1
\end{aligned}
$$

and $\epsilon$ is a sufficiently small, positive nonzero parameter. It may be checked easily that $\rho_{\epsilon}(r)$ is continuous and has continuous first derivatives everywhere. Further, the following estimates hold,

$$
\begin{aligned}
& \left|F_{\epsilon}(x)\right| \leq k(\epsilon) x \text { for } 0 \leq x \leq 1 \\
& \left|F_{\epsilon}(x)\right| \leq k(\epsilon) \text { for } 1<x<\infty \\
& \left|F_{\epsilon}^{\prime}(x)\right| \leq k(\epsilon) \text { for } 0 \leq x<\infty
\end{aligned}
$$

where

$$
k(\epsilon) \equiv \frac{2}{2-\epsilon}>0 .
$$

For the map $\vec{N}(\vec{n})$ with winding number $l$ we choose $l$ nonintersecting geodesic discs on the base $S^{2}$ such that $\vec{N}$ varies smoothly from $\vec{N}=(0,0,1)$ in the center of each disc to $\vec{N}=(0,0,-1)$ at the boundary of each disc and remains at this value in between the discs. The geodesic radius $\vartheta_{0}$ of these geodesic discs has to be chosen sufficiently small so that it is possible to put $l$ nonintersecting discs with this geodesic radius on the base $S^{2}$. We prove in Appendix B that the choice

$$
\vartheta_{0}=\frac{\pi}{4 \sqrt{l}}
$$

is sufficient. Next we give an explicit expression for the map $\vec{N}$ for one geodesic disc which is chosen symmetric about the north pole. With

$$
\vec{N}=(\sin \chi \cos \sigma, \sin \chi \sin \sigma, \cos \chi)
$$

and

$$
\vec{n}=(\sin \vartheta \cos \phi, \sin \vartheta \sin \phi, \cos \vartheta)
$$


we get

$$
\chi(\vartheta)=\pi F_{\epsilon}(\tilde{\vartheta}), \quad \tilde{\vartheta} \equiv \frac{\vartheta}{\vartheta_{0}}
$$

and

$$
\sigma=\phi
$$

where $F_{\epsilon}(\cdot)$ is defined in $(55)$ (with the additional restriction that here $\tilde{\vartheta} \leq$ $4 \sqrt{l}$ because $\vartheta \leq \pi$ ). The above-defined $\vec{N}$ has winding number 1 , and to reach winding number $l$ we have to require that it is nonconstant over $l-1$ further geodesic discs. We do not need, however, explicit expressions for the contribution of these further discs to $\vec{N}$, because their gradients on $S^{2},\left|\nabla^{S^{2}} \vec{N}\right|$ can be estimated by the same majorants as the above explicit expression. The reason for this is that the above symmetrically chosen disc is related to an arbitrary disc with the same geodesic radius by a rotation of the base space $S^{2}$, and the gradient $\left|\nabla^{S^{2}} \vec{N}\right|$ is invariant under such rotations. All that changes is that more regions of the base $S^{2}$ provide nonzero contributions to the gradient.

Next we want to estimate the energy density $\mathcal{E}_{\mathrm{Ni}}(\vec{r}) \sim|\nabla \vec{N}|^{3}$. Here we first estimate (Einstein summation convention is understood, $k, a=1 \ldots 3$ )

$$
|\nabla \vec{N}|^{2}=\left(\nabla_{k} N^{a}\right)^{2}=\chi_{\vartheta}^{2}\left(\nabla_{k} \vartheta\right)^{2}+\sin ^{2} \chi\left(\nabla_{k} \sigma\right)^{2}
$$

where we used (59), and $\chi_{\vartheta} \equiv \partial_{\vartheta} \chi$. The first term may be estimated by

$$
\chi_{\vartheta}^{2} \leq\left(\frac{\pi}{\vartheta_{0}}\right)^{2} k(\epsilon)^{2}
$$

and, with the help of spherical polar coordinates, by

$$
\begin{aligned}
\left(\nabla_{k} \vartheta\right)^{2} & =\vartheta_{r}^{2}+\frac{1}{r^{2}} \vartheta_{\theta}^{2} \\
& =\frac{4 \rho_{r}^{2} \cos ^{2} \rho \sin ^{2} \theta}{1-\sin ^{2} \rho \sin ^{2} \theta}+\frac{\sin ^{2} \rho}{r^{2}} \frac{4 \cos ^{2} \theta}{1-\sin ^{2} \rho \sin ^{2} \theta} \\
& \leq 4 \rho_{r}^{2}+4 \frac{\rho^{2}}{r^{2}} \leq 8 \pi^{2} \frac{k(\epsilon)^{2}}{R^{2}}
\end{aligned}
$$

where we used

$$
\frac{\cos ^{2} \alpha}{1-\sin ^{2} \alpha \sin ^{2} \beta} \leq 1, \quad \sin \alpha \leq \alpha
$$


as well as Eqs. (54) and (56). For the second term we find

$$
\sin ^{2} \chi \leq \sin ^{2}\left(\pi k(\epsilon) \frac{\vartheta}{\vartheta_{0}}\right) \leq\left(\frac{\pi k(\epsilon)}{\vartheta_{0}}\right)^{2} \sin ^{2} \vartheta
$$

where we used

$$
\sin a x \leq a \sin x \quad \text { for } \quad a \geq 1, \quad x \in\left[0, \frac{\pi}{2}\right]
$$

which we prove in Appendix B. Further we find, using $\sigma=\phi$ and the expression for $\phi$ in Eq. (118)

$$
\begin{gathered}
\sin ^{2} \vartheta\left(\nabla_{k} \phi\right)^{2}=4 \sin ^{2} \rho \sin ^{2} \theta\left(1-\sin ^{2} \rho \sin ^{2} \theta\right) . \\
\cdot\left(\frac{1}{r^{2} \sin ^{2} \theta}+\frac{\rho_{r}^{2} \cos ^{2} \theta+\left(1 / r^{2}\right) \cos ^{2} \rho \sin ^{2} \rho \sin ^{2} \theta}{\left(1-\sin ^{2} \rho \sin ^{2} \theta\right)^{2}}\right) \\
=4 \frac{\sin ^{2} \rho}{r^{2}}\left(1-\sin ^{2} \rho \sin ^{2} \theta\right) \\
+4 \sin ^{2} \rho \sin ^{2} \theta \rho_{r}^{2} \frac{\cos ^{2} \theta}{1-\sin ^{2} \rho \sin ^{2} \theta} \\
+4 \sin ^{2} \rho \sin ^{4} \theta \frac{\sin ^{2} \rho}{r^{2}} \frac{\cos ^{2} \rho}{1-\sin ^{2} \rho \sin ^{2} \theta} \\
\leq 4\left(\frac{\pi k(\epsilon)}{R}\right)^{2}+4\left(\frac{\pi k(\epsilon)}{R}\right)^{2}+4\left(\frac{\pi k(\epsilon)}{R}\right)^{2}=12\left(\frac{\pi k(\epsilon)}{R}\right)^{2} .
\end{gathered}
$$

Putting everything together, we therefore find

$$
|\nabla \vec{N}|^{2} \leq 20\left(\frac{\pi^{2} k(\epsilon)^{2}}{\vartheta_{0} R}\right)^{2}=20 l\left(\frac{4 \pi k(\epsilon)^{2}}{R}\right)^{2}
$$

Taking the power of $\frac{3}{2}$ and integrating over the ball $B^{R}$ we estimate the energy by

$$
E_{\mathrm{Ni}}=\int d^{3} r|\nabla \vec{N}|^{3} \leq \int_{B^{R}} d^{3} r 5^{\frac{3}{2}} l^{\frac{3}{2}}\left(\frac{8 \pi k(\epsilon)^{2}}{R}\right)^{3}=\frac{4 \pi}{3} 5^{\frac{3}{2}} l^{\frac{3}{2}}\left(8 \pi k(\epsilon)^{2}\right)^{3}
$$


Finally, for the estimate it is sufficient if the estimating field configuration is a distribution. Therefore we may now perform the $\operatorname{limit}_{\epsilon \rightarrow 0} k(\epsilon)=1$ and get, for $Q=l^{2}$,

$$
E_{\mathrm{Ni}} \leq \frac{4 \pi}{3}(8 \pi)^{3} 5^{\frac{3}{2}} l^{\frac{3}{2}}
$$

In the cases when the Hopf index $Q$ is not a square, we shall find a slightly weaker bound (i.e., a slightly larger value for the constant $C_{2}$ ). Again following [19], we write $Q$ as

$$
Q=l^{2}+m, \quad m \in[1,2 l]
$$

and estimate the energy by the following Hopf map. It maps the ball $B^{R}$ with radius $R$ about the origin to the two-sphere with a contribution of $l^{2}$ to the Hopf index in exactly the way constructed above for the case $Q=l^{2}$. Further, it maps $m$ balls $B_{i}^{1}, i=1 \ldots m$ with unit radius to the $S^{2}$ such that each ball contributes one unit to the Hopf index. Here the $m$ unit balls are chosen such that they intersect neither each other nor the ball $B^{R}$. The map from $B^{R}$ contributes exactly the above-calculated expression (77) to the energy, whereas the $m$ maps from the $B_{i}^{1}$ contribute the energy of a unit Hopf map each. One might think to take just the energy (77) with $l=1$ for each energy contribution, but we can do better. The reason is that we were forced to choose $\vartheta_{0}$ rather small in Eq. (58) to ensure that we can put $l$ geodesic discs on one $S^{2}$. But here we are only interested in a map with winding number one, therefore we can choose the "geodesic disc" equal to the full $S^{2}$, i.e., $\vartheta_{0}=\pi$. This leads to the following energy estimate

$$
E_{\mathrm{Ni}} \leq \frac{4 \pi}{3}(8 \pi)^{3} 5^{\frac{3}{2}} l^{\frac{3}{2}}+\frac{4 \pi}{3}(2 \pi)^{3} 5^{\frac{3}{2}} m
$$

which we want to estimate in terms of $Q$ again, i.e.,

$$
c_{2} l^{\frac{3}{2}}+c_{3} m \leq c_{4}\left(l^{2}+m\right)^{\frac{3}{4}}
$$

Here the 1.h.s. grows linearly in $m$, whereas the r.h.s. grows sublinearly, therefore the inequality certainly holds for all $m \in[1,2 l]$ if it holds for the maximum value $m=2 l$, i.e.,

$$
c_{2} l^{\frac{3}{2}}+2 c_{3} l \leq c_{4}\left(l^{2}+2 l\right)^{\frac{3}{4}} .
$$


With the help of the inequality

$$
c_{2}+2 c_{3} l^{-\frac{1}{2}} \leq c_{2}+2 c_{3} \leq\left(c_{2}+2 c_{3}\right)\left(1+\frac{2}{l}\right)^{\frac{3}{4}}
$$

we see that the above inequality certainly holds for $c_{4}=c_{2}+2 c_{3}$. Inserting the concrete numbers from (79) we therefore get

$$
E_{\mathrm{Ni}} \leq \frac{4 \pi}{3}(2 \pi)^{3}\left(4^{3}+2\right) 5^{\frac{3}{2}} Q^{\frac{3}{4}}
$$

as our final estimate for general $Q$.

\subsection{Upper bounds for the AFZ and Faddeev-Niemi models}

We may use our result to obtain upper bounds for the energies of the AFZ and Faddeev-Niemi models, as well. For the bound on the AFZ model we use the simple inequality for the energy densities

$$
\mathcal{E}_{4} \leq \frac{1}{2} \mathcal{E}_{2}^{2}
$$

which is obvious from the $(\vartheta, \phi)$ parametrization, see Eqs. (119) and (120). It follows immediately that $E_{\mathrm{AFZ}} \leq\left(\frac{1}{2}\right)^{\frac{3}{4}} E_{\mathrm{Ni}}$ and, therefore,

$$
E_{\mathrm{AFZ}} \leq\left(\frac{1}{2}\right)^{\frac{3}{4}} \frac{4 \pi}{3}(2 \pi)^{3}\left(4^{3}+2\right) 5^{\frac{3}{2}} Q^{\frac{3}{4}}
$$

For the Faddeev-Niemi model with energy density $\mathcal{E}_{\mathrm{FN}}=\mathcal{E}_{2}+\lambda \mathcal{E}_{4}$ we consider the case $Q=l^{2}$ of a square Hopf index first. We use Eq. (75) for the density $\mathcal{E}_{2}$ and the inequality (84) and find

$$
\begin{aligned}
E_{\mathrm{FN}} & =\int_{B^{R}} d^{3} r\left(\mathcal{E}_{2}+\lambda \mathcal{E}_{4}\right) \\
& \leq \int_{B^{R}} d^{3} r\left(20 l\left(\frac{4 \pi}{R}\right)^{2}+\frac{\lambda}{2}(20 l)^{2}\left(\frac{4 \pi}{R}\right)^{4}\right) \\
& =\frac{4 \pi}{3} R^{3}\left(20 l\left(\frac{4 \pi}{R}\right)^{2}+\frac{\lambda}{2}(20 l)^{2}\left(\frac{4 \pi}{R}\right)^{4}\right)
\end{aligned}
$$


and, with the choice

$$
R=R_{0} \sqrt{l}
$$

we get

$$
E_{\mathrm{FN}} \leq 20 \frac{4 \pi}{3}(4 \pi)^{2} l^{\frac{3}{2}}\left(R_{0}+10(4 \pi)^{2} \frac{\lambda}{R_{0}}\right) .
$$

For a neat estimate we now minimize the expression in brackets w.r.t. $R_{0}$ which leads to

$$
R_{0}=4 \pi \sqrt{10 \lambda}
$$

and to

$$
E_{\mathrm{FN}} \leq 40 \frac{4 \pi}{3}(4 \pi)^{3} \sqrt{10 \lambda} l^{\frac{3}{2}}
$$

Here we have separated a factor $(4 \pi)^{3}$ because it can be replaced by $\pi^{3}$ for winding number one, exactly as in the estimate for the Nicole model, which we need for general $Q$. Again we write $Q=l^{2}+m$ and estimate

$$
E_{\mathrm{FN}} \leq 40 \frac{4 \pi}{3}(4 \pi)^{3} \sqrt{10 \lambda} l^{\frac{3}{2}}+40 \frac{4 \pi}{3} \pi^{3} \sqrt{10 \lambda} m
$$

and finally by

$$
E_{\mathrm{FN}} \leq 40 \frac{4 \pi}{3} \pi^{3} \sqrt{10 \lambda}\left(4^{3}+2\right) Q^{\frac{3}{4}}
$$

in complete analogy to the Nicole model.

\section{Conclusions}

Combining numerical and analytical techniques, we found the energies for static solutions of the Nicole model within the symmetric ansatz (8) for a wide (in principle arbitrary) range of the integers $m$ and $n$. It turns out that the energies of these symmetric solutions grow linearly with the Hopf index $Q=m n$ within a very good accuracy. Together with the sublinear growth inequality of Section $4, E \leq C_{2} Q^{\frac{3}{4}}$ this implies that, for sufficiently large values of $Q$, the symmetric solutions we found cannot be global minima within their respective topological sectors with fixed Hopf index $Q$. It is of some interest to compare this result with the corresponding results for the AFZ and Faddeev-Niemi models. 
For the AFZ model all solutions for the symmetric ansatz (8) and their corresponding energies can be calculated exactly [8]. The energies are

$$
\begin{aligned}
E_{\mathrm{AFZ}} & =8 \sqrt{2} \pi^{2} \sqrt{|m||n|(|m|+|n|)} \\
& =8 \sqrt{2} \pi^{2} Q^{\frac{3}{4}} \sqrt{\mu+\frac{1}{\mu}}, \quad \mu^{2} \equiv\left|\frac{m}{n}\right|
\end{aligned}
$$

and, for $m=n(\mu=1)$ perfectly fit within the upper bound $E_{\mathrm{AFZ}} \leq C_{2} Q^{\frac{3}{4}}$ which holds also for the AFZ model (see Section 4). It is, therefore, plausible to conjecture that these solutions might be true minima for $m=n$ (and, maybe, even for $m \neq n$ provided that $m$ and $n$ do not differ too much).

For the Faddeev-Niemi model the ansatz (8) is not compatible with the equations of motion (due to the lack of conformal symmetry of the latter), but the cylindrically symmetric ansatz

$$
u=f(\eta, \xi) e^{i m \varphi}
$$

for complex $f$ is compatible and leads to an equation in the two independent variables $\eta$ and $\xi$. Numerical investigation of the full e.o.m. in three variables shows that the solutions for the ansatz (94) are true minima only for the lowest values $Q=1,2$ of the Hopf index [4]. In this respect, therefore, the Nicole model bears more similarity with the Faddeev-Niemi model than with the AFZ model. It is tempting to speculate at this instant that the different behaviour of the AFZ model - i.e. the possibility that the symmetric solutions (93) are true minima for arbitrary $Q$ - is related to the integrability properties of the latter. But at the moment this is, of course, only a speculation which deserves further investigation.

\section{Appendix A: Facts about Hopf maps}

A Hopf map is a map from the three-sphere $S^{3}$ to the two-sphere $S^{2}$. The third homotopy group of the two-sphere is nontrivial, $\Pi_{3}\left(S^{2}\right)=\mathbb{Z}$, therefore Hopf maps are characterised by an integer topological index, the so-called Hopf index $Q$. The three-sphere is topologically equivalent to one-point compactified three-dimensional Euclidean space $\mathbb{R}_{0}^{3}$, therefore, for each Hopf map $S^{3} \rightarrow S^{2}$ there exists a corresponding map $\mathbb{R}_{0}^{3} \rightarrow S^{2}$, which we shall call Hopf 
map, as well, and which has the same Hopf index (due to the metric independence of a topological index). Explicitly, a Hopf map $\mathbb{R}_{0}^{3} \rightarrow S^{2}$ may be given by a three-component unit vector field

$$
\vec{n}(\vec{r}): \mathbb{R}_{0}^{3} \rightarrow S^{2}, \quad \vec{n}^{2}=1, \quad \lim _{\vec{r} \rightarrow \infty}=\vec{n}_{0}=\mathrm{const}
$$

where the tip of the unit vector field spans the unit two-sphere, or via stereographic projection

$$
\vec{n}=\frac{1}{1+|u|^{2}}(u+\bar{u},-i(u-\bar{u}), 1-u \bar{u}) ; \quad u=\frac{n_{1}+i n_{2}}{1+n_{3}} .
$$

by a complex field

$$
u(\vec{r}): \mathbb{R}_{0}^{3} \rightarrow \mathbb{C}_{0}, \quad \lim _{\vec{r} \rightarrow \infty}=u_{0}=\text { const }
$$

where our conventions are such that the projection is from the south pole to the equatorial plane of the two-sphere. Further, $\mathbb{C}_{0}$ is the one-point compactified complex plane. A third possibility to parametrize a Hopf map is by the spherical angles $\phi \in[0,2 \pi], \vartheta \in[0, \pi]$, which are related to the unit vector $\vec{n}$ and complex field $u$ via

$$
u=\tan \frac{\vartheta}{2} e^{i \phi}, \quad \vec{n}=(\sin \vartheta \cos \phi, \sin \vartheta \sin \phi, \cos \vartheta) .
$$

The geometry behind the Hopf map may be visualised as follows. The pre-images under the inverse of the Hopf map $u$ of points in the target space $S^{2}$ (or, equivalently, $\mathbb{C}_{0}$ ) are closed curves in $\mathbb{R}_{0}^{3}$ (in general, knots), where each two curves corresponding to two different points in target space are linked exactly $Q$ times. An analytic expression for the Hopf index $Q$ is

$$
Q=\frac{1}{16 \pi^{2}} \int d^{3} r \overrightarrow{\mathcal{A}} \cdot \overrightarrow{\mathcal{B}}
$$

where $\overrightarrow{\mathcal{B}}$ is the Hopf curvature

$$
\overrightarrow{\mathcal{B}}=\frac{2}{i} \frac{\nabla u \times \nabla \bar{u}}{(1+u \bar{u})^{2}}=-\frac{1}{2} \epsilon^{a b c} n^{a} \nabla n^{b} \times \nabla n^{c}=-\sin \vartheta \nabla \vartheta \times \nabla \phi
$$

and $\overrightarrow{\mathcal{A}}$ is the gauge potential for the "magnetic field" $\overrightarrow{\mathcal{B}}, \overrightarrow{\mathcal{B}}=\nabla \times \overrightarrow{\mathcal{A}}$. There is no local expression for $\overrightarrow{\mathcal{A}}$ in terms of the Hopf map $u$ alone (however, there 
certainly exist non-local expressions for certain gauges, as is obvious from the analogy with electrodynamics, by choosing, e.g., the Couloumb gauge $\nabla \cdot \overrightarrow{\mathcal{A}}=0$ which implies $\left.\overrightarrow{\mathcal{A}}=\nabla \times \int d^{3} r^{\prime} \Delta^{-1}\left(\vec{r}-\vec{r}^{\prime}\right) \overrightarrow{\mathcal{B}}\left(\vec{r}^{\prime}\right)\right)$.

Geometrically, $\overrightarrow{\mathcal{B}}$ is the Hodge dual of the pullback of the area two-form on $S^{2}$ under the Hopf map $u$, i.e.,

$$
\begin{gathered}
\mathcal{B} \equiv \mathcal{B}_{k} d r^{k}=* \mathcal{F}, \\
\mathcal{F} \equiv \frac{1}{2} \mathcal{F}_{j k} d r^{j} d r^{k}, \quad \mathcal{B}_{k}=\varepsilon_{k l m} \mathcal{F}_{l m}
\end{gathered}
$$

where

$$
\mathcal{F}=u^{*}(\Omega), \quad \Omega=\frac{2}{i} \frac{d \zeta d \bar{\zeta}}{(1+\zeta \bar{\zeta})^{2}}, \quad \int_{S^{2}} \Omega=4 \pi .
$$

The second cohomology group of the three-sphere is trivial, $H_{2}\left(S^{3}\right)=0$, therefore the closed two-form $\mathcal{F}$ must be exact, $\mathcal{F}=d \mathcal{A}$. It follows that the gauge potential $\overrightarrow{\mathcal{A}}$ is globally well-defined for appropriately chosen gauges (e.g., in the Couloumb gauge).

The simplest Hopf map with Hopf index 1 is

$$
u=i \frac{2(x+i y)}{2 z+i\left(r^{2}-1\right)}
$$

(the irrelevant factor $i$ has been introduced to be in exact agreement with the simplest Hopf map of Eq. (12)). Its level curves (i.e., the pre-images of points $u=$ const) are circles which lie on tori, and each two different circles are linked precisely once.

There are different ways to construct Hopf maps explicitly. One method which we shall need is to compose a given Hopf map with a map $S^{2} \rightarrow S^{2}$,

$$
\vec{N}(\vec{n}(\vec{r})): \mathbb{R}_{0}^{3} \stackrel{\vec{n}(\vec{r})}{\rightarrow} S^{2} \stackrel{\vec{N}(\vec{n})}{\rightarrow} S^{2}
$$

If the Hopf map $\vec{n}(\vec{r})$ has Hopf index $Q$ and the map $\vec{N}(\vec{n})$ has winding number $w$, then the composition map $\vec{N}(\vec{n}(\vec{r}))$ has Hopf index $Q^{\prime}=w^{2} Q$.

A method to construct all possible Hopf maps starts from maps $S^{3} \rightarrow S^{3}$ (or, equivalently, maps $\mathbb{R}_{0}^{3} \rightarrow S^{3}$ ). The third homotopy group of $S^{3}$ is nontrivial, $\Pi_{3}\left(S^{3}\right)=\mathbb{Z}$, therefore such maps are classified by a topological index, the winding number $W$. It is possible to construct Hopf maps $\mathbb{R}_{0}^{3} \rightarrow$ 
$S^{2}$ from maps $\mathbb{R}_{0}^{3} \rightarrow S^{3}$ such that the Hopf index equals the winding number. Explicitly a map $\mathbb{R}_{0}^{3} \rightarrow S^{3}$ may be given by a four component unit vector field

$$
\begin{gathered}
\left(e_{1}(\vec{r}), e_{2}(\vec{r}), e_{3}(\vec{r}), e_{4}(\vec{r})\right): \mathbb{R}_{0}^{3} \rightarrow S^{3}, \quad e_{\alpha} e_{\alpha}=1, \quad \alpha=1, \ldots, 4 \\
\lim _{\vec{r} \rightarrow \infty} e_{\alpha}=e_{\alpha}^{0}=\mathrm{const}
\end{gathered}
$$

then the corresponding Hopf map is given in terms of $u$ as

$$
u=\frac{e_{1}+i e_{2}}{e_{3}+i e_{4}}
$$

or in terms of $\vec{n}$ as

$$
\vec{n}=\left(2 e_{1} e_{3}+2 e_{2} e_{4},-2 e_{1} e_{4}+2 e_{2} e_{3}, e_{3}^{2}+e_{4}^{2}-e_{1}^{2}-e_{2}^{2}\right) .
$$

Further, it is now possible to give an explicit, local expression for the gauge potential $\overrightarrow{\mathcal{A}}$, in terms of the $e_{\alpha}$, as

$$
\overrightarrow{\mathcal{A}}=\frac{2}{i}\left[\left(e_{1}-i e_{2}\right) \nabla\left(e_{1}+i e_{2}\right)+\left(e_{3}-i e_{4}\right) \nabla\left(e_{3}+i e_{4}\right)\right] .
$$

It may be checked without difficulty that indeed $\nabla \times \overrightarrow{\mathcal{A}}=\overrightarrow{\mathcal{B}}$ where $\overrightarrow{\mathcal{B}}$ is the Hopf curvature (100).

As the group manifold of $S U(2)$ is equivalent to the three-sphere $S^{3}$, we may use maps $\mathbb{R}_{0}^{3} \rightarrow S U(2)$ instead. Indeed, with the group element

$$
\begin{gathered}
U=e_{4}-i \vec{e} \cdot \vec{\sigma} \equiv \exp [-i \rho \vec{k} \cdot \vec{\sigma}], \quad \lim _{\vec{r} \rightarrow \infty} U=U_{0}=\mathrm{const} \\
e_{4}=\cos \rho, \quad \vec{e}=\sin \rho \vec{k}, \quad \vec{k}^{2}=1
\end{gathered}
$$

(where $\vec{e}=\left(e_{1}, e_{2}, e_{3}\right)$ and $\vec{\sigma}$ are the Pauli matrices) the Hopf map (109) may be expressed like

$$
\vec{n}=\frac{1}{2} \operatorname{tr} \sigma_{3} U^{\dagger} \vec{\sigma} U
$$

This latter representation may be used to produce ansaetze for Hopf maps with cylindrical symmetry from maps $\mathbb{R}_{0}^{3} \rightarrow S U(2)$ with rotational symmetry. Indeed, for

$$
\vec{k}=\hat{r} \equiv \vec{r} / r, \quad \rho=\rho(r), \quad \rho(0)=0, \quad \lim _{r \rightarrow \infty} \rho=\pi W, \quad W \in \mathbb{Z}
$$


(the conditions on $\rho(r)$ are to ensure a well-defined $U$ on all $\mathbb{R}_{0}^{3}$, and they provide, at the same time, a winding number equal to $W$ for the corresponding map $\mathbb{R}_{0}^{3} \rightarrow S^{3}$ or $\left.\mathbb{R}_{0}^{3} \rightarrow S U(2)\right)$, the resulting group element $U(\rho(r), \hat{r})$ is rotationally invariant in the sense that any rotation in $\mathbb{R}_{0}^{3}$ can be compensated by a $S U(2)$ transformation,

$$
U(\rho(r), O \hat{r}) \equiv \cos \rho(r)-i \sin \left(\rho(r) \vec{\sigma} \cdot(O \hat{r})=V^{\dagger} U(\rho(r), \hat{r}) V\right.
$$

where $O$ is a rotation matrix acting on $\hat{r}$ and $V$ is the $S U(2)$ matrix corresponding to the rotation $O$. Within this ansatz, and using spherical polar coordinates $\hat{r}=(\sin \theta \cos \varphi, \sin \theta \sin \varphi, \cos \theta)$, we get for the complex field $u$

$$
u=\frac{\sin \rho \sin \theta e^{i \varphi}}{\sin \rho \cos \theta+i \cos \rho}
$$

which has cylindrical symmetry in the sense that any rotation $\varphi \rightarrow \varphi+\varphi_{0}$ can be compensated by a phase transformation $u \rightarrow e^{i \varphi_{0}} u$. Further, $u$ is a Hopf map with Hopf index equal to the winding number $W$. For technical reasons we prefer to use a $u$ which is multiplied by $-i$ in our main calculation, i.e.,

$$
u=-i \frac{e_{1}+i e_{2}}{e_{3}+i e_{4}}=\frac{\sin \rho \sin \theta}{\sqrt{\sin ^{2} \rho \cos ^{2} \theta+\cos ^{2} \rho}} e^{i \varphi+i \arctan [\tan \rho \cos \theta]} .
$$

With $u=\tan (\vartheta / 2) e^{i \phi}$ this leads to

$$
\sin \vartheta=2 \sin \rho \sin \theta \sqrt{1-\sin ^{2} \rho \sin ^{2} \theta}, \quad \phi=\varphi+\arctan [\tan \rho \cos \theta] .
$$

Finally, we display the energy densities of the Nicole and AFZ models in the three different parametrizations, $\mathcal{E}_{\mathrm{Ni}}=\mathcal{E}_{2}^{\frac{3}{2}}$ with

$$
\mathcal{E}_{2}=|\nabla \vec{n}|^{2} \equiv\left|\nabla_{k} n^{a}\right|^{2}=4 \frac{\nabla u \cdot \nabla \bar{u}}{(1+u \bar{u})^{2}}=(\nabla \vartheta)^{2}+\sin ^{2} \vartheta(\nabla \phi)^{2}
$$

and $\mathcal{E}_{\mathrm{AFZ}}=\mathcal{E}_{4}^{\frac{3}{4}}$ with

$$
\mathcal{E}_{4}=\overrightarrow{\mathcal{B}}^{2}=\frac{1}{2}\left(\left(\nabla n^{b} \cdot \nabla n^{b}\right)^{2}-\left(\nabla n^{b} \cdot \nabla n^{c}\right)^{2}\right)=
$$




$$
4 \frac{(\nabla u \cdot \nabla \bar{u})^{2}-(\nabla u)^{2}(\nabla \bar{u})^{2}}{(1+u \bar{u})^{4}}=\sin ^{2} \vartheta\left((\nabla \vartheta)^{2}(\nabla \phi)^{2}-(\nabla \vartheta \cdot \nabla \phi)^{2}\right) .
$$

\section{Appendix B}

\section{B1: Proof that}

$$
(a+b)^{\frac{3}{2}} \geq c a^{\frac{1}{2}} b \quad \text { for } \quad a, b \geq 0, \quad c \leq \sqrt{3} \frac{3}{2} .
$$

By squaring the above expression and subtracting the r.h.s. we get

$$
a^{3}+3 a^{2} b+3\left(1-d^{2}\right) a b^{2}+b^{3} \geq 0, \quad d^{2} \equiv \frac{c^{2}}{3}
$$

or, by introducing the variable $x=\frac{a}{b}$,

$$
h(x) \equiv x^{3}+3 x^{2}+3\left(1-d^{2}\right) x+1 \geq 0 \quad \text { for } \quad x \geq 0 .
$$

This problem we solve by first calculating the minimum $x_{\min }$ of $h(x)$ for $x \geq 0$, and by then requiring that $h\left(x_{\min }\right)=0$. The minimum is at

$$
h^{\prime}(x)=3\left(x^{2}+2 x+\left(1-d^{2}\right)\right) \equiv 0 \quad \Rightarrow \quad x_{\min }=d-1
$$

and the condition $h\left(x_{\min }\right)=0$ finally leads to

$$
h\left(x_{\text {min }}\right)=d^{2}(3-2 d) \equiv 0 \quad \wedge \quad d \geq 1 \quad \Rightarrow \quad d=\frac{3}{2}
$$

which implies (121).

QED

B2: Proof that one may cover the unit sphere with $l$ nonintersecting discs with geodesic radius $\vartheta_{0}=\frac{\pi}{4 \sqrt{l}}$.

First we study the case where $l$ is a square, $l=k^{2}$. We divide the northern hemisphere of the two-sphere into segments by circles which are concentric about the north pole and are given by

$$
\vartheta=\frac{m \pi}{2 k}, \quad m=1 \ldots k .
$$


This divides the northern hemisphere into one disc with geodesic radius $\frac{\pi}{2 k}$ about the north pole (on which one geodesic disc with geodesic radius $\vartheta_{0}=\frac{\pi}{4 k}$ certainly does fit) and into sphere segments such that the boundary circles have a geodesic distance of $\frac{\pi}{2 k}$. Therefore discs with geodesic diameter $2 \vartheta_{0}=$

$\frac{\pi}{2 k}$ exactly fit in between. Further, $2 m+1$ geodesic discs fit on the segment between the circles at $\vartheta=\frac{m \pi}{2 k}$ and at $\vartheta=\frac{(m+1) \pi}{2 k}$. This is because the central circle of the segment has a circumference wich is bigger than the sum of $m+1$ diameters of the geodesic discs, i.e.,

$$
2 \pi \sin \left(\left(m+\frac{1}{2}\right) \frac{\pi}{2 k}\right) \geq 2 \pi \frac{2}{\pi}\left(\left(m+\frac{1}{2}\right) \frac{\pi}{2 k}\right)=(2 m+1) \frac{\pi}{k} \geq(2 m+1) \frac{\pi}{2 k}
$$

where we used

$$
\sin x \geq \frac{2}{\pi} x \quad \text { for } \quad x \in\left[0, \frac{\pi}{2}\right]
$$

Finally, summing over all discs on all segments (including the first disc at the segment which is itself a disc) we find

$$
\sum_{m=0}^{k-1}(2 m+1)=\sum_{m=1}^{k}(2 m-1)=k^{2}
$$

therefore we may indeed distribute $k^{2}$ nonintersecting geodesic discs with geodesic radius $\vartheta_{0}=\frac{\pi}{4 k}$ on the northern hemisphere.

It remains to study the case where $l$ is not a square. Then we write $l=k^{2}+j$. We distribute the $k^{2}$ discs on the northern hemisphere as just described. Further, we distribute the remaining $j$ discs on the southern hemisphere. This is obviously possible as long as $k^{2} \geq j$. So we cover all cases except the case $l=3$. This latter case may be proven easily with the help of elementary geometry.

QED

\section{B3: Proof that}

$$
\sin a x \leq a \sin x \quad \text { for } \quad a \geq 1, \quad x \in\left[0, \frac{\pi}{2}\right]
$$

We introduce $a \equiv 1+\epsilon, \epsilon \geq 0$ and write

$$
\sin ((1+\epsilon) x)=\sin x \cos \epsilon x+\cos x \sin \epsilon x
$$




$$
\leq \sin x+\epsilon x \cos x \leq \sin x+\epsilon \sin x \equiv a \sin x
$$

where we used $\cos x \leq 1, \sin x \leq x$ for $x \geq 0$, and

$$
x \cos x \leq \sin x \quad \Rightarrow \quad \tan x \geq x \quad \text { for } \quad x \in\left[0, \frac{\pi}{2}\right]
$$

which is obviously true.

QED

\section{Appendix C: \\ On the possibility of a lower bound $C_{1} Q^{\frac{3}{4}} \leq E_{Q}$}

It may be useful to briefly comment on the problem of deriving a lower bound for the energy of the type $C_{1} Q^{\frac{3}{4}} \leq E_{Q}$, analogously to the case of the Faddeev-Niemi model, where this bound can be proven (VakulenkoKapitansky bound, see e.g. [20], [21], [22]). In the case of the Faddeev-Niemi model, the first step in the proof is Hoelder's inequality (we use the conventions of [23], where all inequalities of this appendix can be found; further, we assume that $\overrightarrow{\mathcal{A}}$ and $\overrightarrow{\mathcal{B}}$ belong to the appropriate Sobolev spaces such that all integrals below exist)

$$
\begin{gathered}
16 \pi^{2} Q \equiv \int d^{3} r \overrightarrow{\mathcal{A}} \cdot \overrightarrow{\mathcal{B}} \leq\left(\int d^{3} r|\overrightarrow{\mathcal{A}}|^{p}\right)^{\frac{1}{p}}\left(\int d^{3} r|\overrightarrow{\mathcal{B}}|^{q}\right)^{\frac{1}{q}} \\
\frac{1}{p}+\frac{1}{q}=1 .
\end{gathered}
$$

For the Faddeev-Niemi model one has to choose $p=6, q=\frac{6}{5}$ and may estimate the first term with the help of the Gagliardo-Nirenberg-Sobolev (GNS) inequality,

$$
\left(\int d^{3} r|\overrightarrow{\mathcal{A}}|^{6}\right)^{\frac{1}{6}} \leq c\left(\left.\int d^{3} r|\nabla| \overrightarrow{\mathcal{A}}\right|^{2}\right)^{\frac{1}{2}}
$$

(here and below $c$ is an unspecified constant) and, when the Couloumb gauge condition $\nabla \cdot \overrightarrow{\mathcal{A}}=0$ is imposed, the integrand can be reexpressed like

$$
|\nabla| \overrightarrow{\mathcal{A}}||^{2} \leq c\left|\sum_{j k} \partial_{j} \mathcal{A}_{k}\right|^{2}=c\left(|\nabla \times \overrightarrow{\mathcal{A}}|^{2}+\nabla \cdot \vec{\Lambda}\right)
$$


where

$$
\Lambda_{1}=\left(\mathcal{A}_{2} \partial_{2}+\mathcal{A}_{3} \partial_{3}\right) \mathcal{A}_{1}-\mathcal{A}_{1}\left(\partial_{2} \mathcal{A}_{2}+\partial_{3} \mathcal{A}_{3}\right)
$$

and $\Lambda_{2}, \Lambda_{3}$ follow by permutation. Therefore, the integrand is the sum of $\overrightarrow{\mathcal{B}}^{2}$ and a total divergence which does not contribute to the integral (for $\overrightarrow{\mathcal{A}}$ which decay sufficiently fast in the limit $|\vec{r}| \rightarrow \infty$; this is automatically satisfied by the class of $\overrightarrow{\mathcal{A}}$ we consider). The proof continues with the interpolation inequality

$$
\left(\int d^{3} r|\overrightarrow{\mathcal{B}}|^{\frac{6}{5}}\right)^{\frac{5}{6}} \leq\left(\int d^{3} r|\overrightarrow{\mathcal{B}}|\right)^{\frac{2}{3}}\left(\int d^{3} r|\overrightarrow{\mathcal{B}}|^{2}\right)^{\frac{1}{6}}
$$

for the second term. This leads to

$$
16 \pi^{2} Q \leq c\left(\int d^{3} r|\overrightarrow{\mathcal{B}}|^{2}\right)^{\frac{2}{3}}\left(\int d^{3} r|\overrightarrow{\mathcal{B}}|\right)^{\frac{2}{3}}
$$

and, together with $|\overrightarrow{\mathcal{B}}|^{2}=\mathcal{E}_{4}$ and $|\overrightarrow{\mathcal{B}}| \leq \frac{1}{\sqrt{2}} \mathcal{E}_{2}$, to

$$
\begin{aligned}
16 \pi^{2} Q & \leq c \lambda^{-\frac{2}{3}}\left[\left(\lambda \int d^{3} r \mathcal{E}_{4}\right)^{\frac{1}{2}}\left(\int d^{3} r \mathcal{E}_{2}\right)^{\frac{1}{2}}\right]^{\frac{4}{3}} \\
& \leq c \lambda^{-\frac{2}{3}}\left[\lambda \int d^{3} r \mathcal{E}_{4}+\int d^{3} r \mathcal{E}_{2}\right]^{\frac{4}{3}}
\end{aligned}
$$

where we used Cauchy's inequality in the last step. The Vakulenko-Kapitansky bound follows immediately by taking the above inequality to the power $\frac{3}{4}$.

Now let us point out where the analogous proof fails for the AFZ and Nicole models. One again starts with Hoelder's inequality (133), but now one would have to choose $p=3$ and $q=\frac{3}{2}$. With the GNS inequality one can again estimate the first term

$$
\left(\int d^{3} r|\overrightarrow{\mathcal{A}}|^{3}\right)^{\frac{1}{3}} \leq c\left(\int d^{3} r|\nabla| \overrightarrow{\mathcal{A}}||^{\frac{3}{2}}\right)^{\frac{2}{3}}
$$

but now the integrand is

$$
|\nabla| \overrightarrow{\mathcal{A}}||^{\frac{3}{2}} \leq c\left|\sum_{j k} \partial_{j} \mathcal{A}_{k}\right|^{\frac{3}{2}}=c\left(|\nabla \times \overrightarrow{\mathcal{A}}|^{2}+\nabla \cdot \vec{\Lambda}\right)^{\frac{3}{4}}
$$

and therefore no longer a total derivative, and we cannot express the integrand in terms of the Hopf curvature alone. We have not been able to 
overcome this difficulty. For our main results, however, the upper bound is more important, which shows that for sufficiently high Hopf index our solutions of Section 2 cannot be true minimizers of the energy.

\section{Acknowledgement:}

This research was partly supported by MCyT(Spain) and FEDER (FPA200501963), Incentivos from Xunta de Galicia and the EC network "EUCLID". Further, we thank "Centro de computacion de Galicia" (CESGA) for computer support. CA acknowledges support from the Austrian START award project FWF-Y-137-TEC and from the FWF project P161 05 NO 5 of N.J.

Mauser. AW gratefully acknowledges support from the Polish Ministry of Education and Science.

\section{References}

[1] L.D. Faddeev, in "40 Years in Mathematical Physics", World Scientific, Singapore 1995.

[2] L.D. Faddeev and A.J. Niemi, Nature 387 (1997) 58; hep-th/9610193.

[3] J. Gladikowski and M. Hellmund, Phys. Rev. D56 (1997) 5194; hepth/9609035.

[4] R.A. Battye and P. Sutcliffe, Proc. Roy. Soc. Lond. A455 (1999) 4305, hep-th/9811077.

[5] R.A. Battye and P. Sutcliffe, Phys. Rev. Lett. 81 (1998) 4798.

[6] J. Hietarinta and P. Salo, Phys. Rev. D62 (2000) 081701.

[7] H. Aratyn, L.A. Ferreira, and A. Zimerman, Phys. Lett. B456 (1999) 162.

[8] H. Aratyn, L.A. Ferreira, and A. Zimerman, Phys. Rev. Lett. 83 (1999) 1723 .

[9] O. Babelon and L.A. Ferreira, JHEP 0211 (2002) 020. 
[10] L.A. Ferreira, hep-th/0601235.

[11] D.A. Nicole, J. Phys. G4 (1978) 1363.

[12] C. Adam and J. Sanchez-Guillen, JHEP01 (2005) 004.

[13] A. Wereszczyński, Eur. Phys. J. C 41 (2005) 265.

[14] C. Adam, J. Sanchez-Guillen, and A. Wereszczyński, hep-th/0511277; to appear in J. Math. Phys.

[15] O. Alvarez, L.A. Ferreira, and J. Sánchez-Guillén, Nucl. Phys. B 529, 689 (1998).

[16] C. Adam and J. Sanchez-Guillen, Phys. Lett. B 626 (2005) 235.

[17] A. Wereszczyński, Phys. Lett. B 621 (2005) 201.

[18] C. Adam, J. Math. Phys. 45 (2004) 4017.

[19] F. Lin and Y. Yang, Comm. Math. Phys. 249 (2004) 273.

[20] A. Vakulenko and L. Kapitansky, Sov. Phys. Doklady 24 (1979) 433.

[21] R.S. Ward, Nonlinearity 12, 241 (1999).

[22] S.V. Shabanov, J. Math. Phys. 43 (2002) 4127.

[23] L.C. Evans, "Partial Differential Equations", Graduate Studies in Mathematics, Vol. 19; American Mathematical Society, Providence, Rhode Island 1998. 


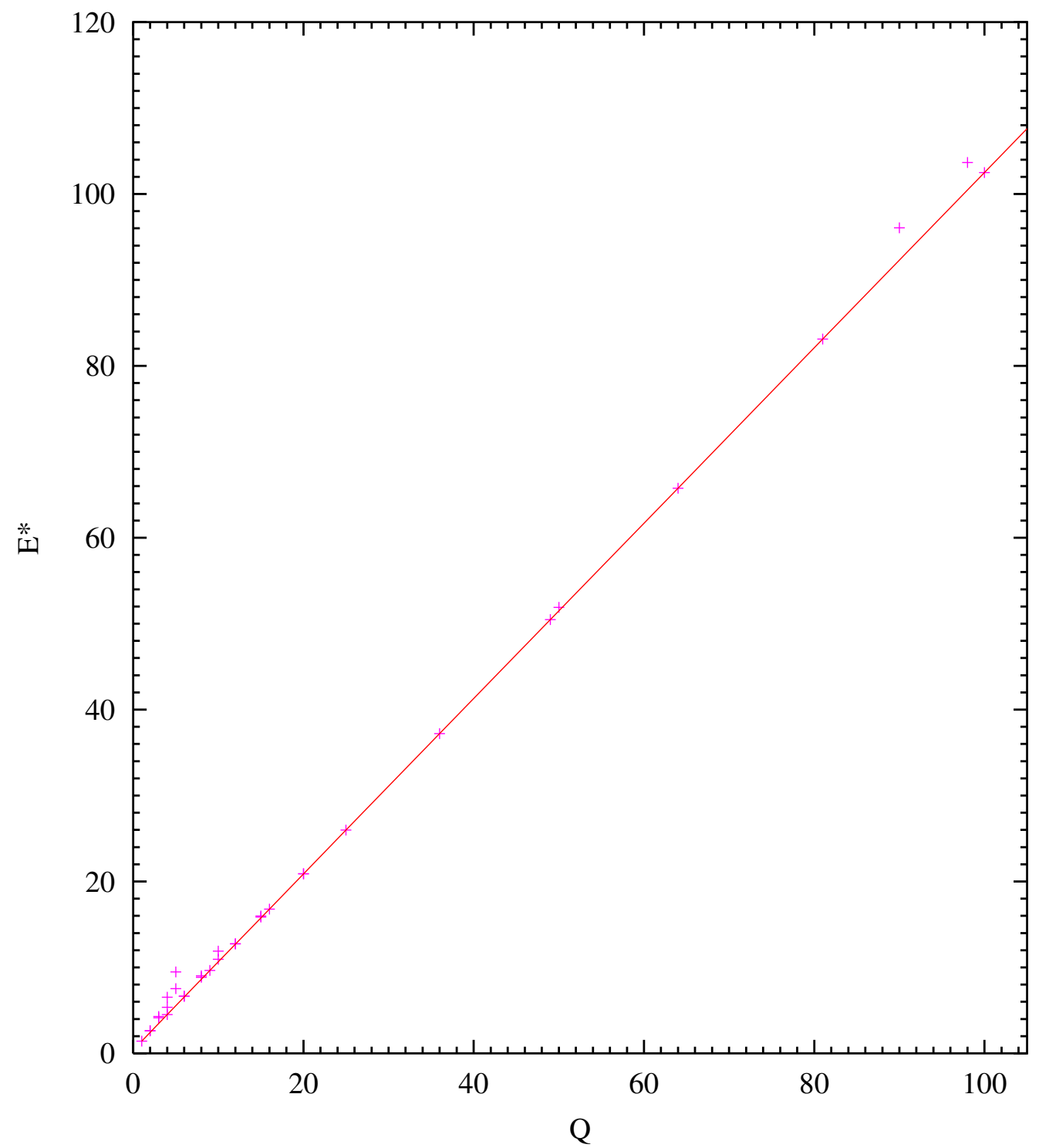

Figure 1: The rescaled energies $E^{*}=\left(32 \pi^{2}\right)^{-1} E$ (vertical axis) are plotted versus the Hopf index $Q=m n$. The straight line connects energies for $m=n$. The energies for $m \neq n$ lie slightly above this line. 